\title{
Direct Cost Estimation of Infection Control Strategy in Pediatric Intensive Care Unit at Tanta University Hospital
}

\author{
Mohammed Nagy Hammad Elganiny ${ }^{1}$, Ahmed Abd Elbasset Abo Elezz ${ }^{1}$, Enas Arafa Elzimmrany ${ }^{2}$ \\ and Dina Hassan Abd Elhady ${ }^{3}$
}

Department of Pediatric Medicine ${ }^{1}$ and Department of Clinical Pathology ${ }^{2}$, Faculty of Medicine, Department of Applied Statistics Faculty of Commerce ${ }^{3}$ - Tanta University, Egypt Corresponding author: Mohammed Nagy Hammad Elganiny; Mobile: 01066802698;

Email: greeneyesnagy86@gmail.com

\section{ABSTRACT}

Background: Hospital-acquired infections (HAIs) are a major complication of hospital care in adult and children. Estimating the cost of infection control is important because HAIs cost a lot by extending patient length of stay this is a major risk factor for HAI.

Objectives: Estimation of the direct cost of medical infection control measures and the direct cost of hospitalization among patients with and without hospital-acquired infections in the Pediatric Intensive Care Unit at Tanta University Hospital.

Subjects and Methods: fifty infants and children (with and without HAI) who were admitted to PICU were subjected to clinical examination and investigation to diagnose infection. The direct cost included feeding requirements, investigations, procedures, medications and infection control measures. Prices used according to the period from October 2016 to September 2017 for calculating patients cost.

Results: The average total direct cost of hospitalization, for cases with HAI was $8940.45 \mathrm{EP}$, which was significantly higher than that for patients without HAI (2935.63 EP). There was a significant difference in the average cost per day between patients with and without HAI. The average total direct cost of management for cases with HAI was 7068.77 E.P, which was significantly higher than that for patients without HAI (2191.66 E.P). The average total infection control cost for cases with HAI was 1871.69 E.P, which was significantly higher than that for cases without HAI (743.96 E.P). The mean length of stay (LOS) in PICU was significantly longer for patients with HAI (19.08 days), while in patients without HAI was 7.44 days (range 4-11).

Conclusion: The cost of infection control is much lower than the cost of hospital-acquired infections management that prolongs hospital stay causing financial losses to the health system.

Key words: Cost Estimation; Infection Control Strategy; Pediatric Intensive Care Unit.

\section{INTRODUCTION}

Healthcare-associated infections (HAIs) in the Pediatric Intensive Care Units (PICUs) are a major problem resulting in a prolonged length of hospital stay, increased medical costs, and increased morbidity and mortality ${ }^{(1)}$.

Blood stream infection (BSI), urinary tract infection (UTI) and pneumonia are the most common NI reported from an Intensive Care Unit (ICU) set-up, and associated with increased length of hospital stay, health-care expenditure, morbidity and mortality ${ }^{(2)}$.

The level of risk relates to the type of clinical contact healthcare workers who are dealing with potentially infected or colonized patient groups, instruments or environments, and the health status of the healthcare worker (e.g. immunized or immuno-compromized) ${ }^{(3)}$.

Vascular access is crucial in the critical care setting. Central venous catheters (CVCs), peripherally inserted central catheters (PICCs), and arterial catheters, are common place in 
pediatric critical care units ${ }^{(4)}$.

Ventilator-associated pneumonia (VAP) is the second most common hospital acquired infection in the PICU after bacteremia ${ }^{(5)}$. Large pediatric studies are limited, but in 2002 a prospective study showed that approximately $5 \%$ of mechanically ventilated children in the PICU developed VAP and $20 \%$ of those died ${ }^{(6)}$. Due to awareness and implementation of prevention measures, VAP incidence density within the PICU has declined from 5.9/1,000-ventilator days in 1997 to a pooled mean of $0.7-1.1 / 1,000$ ventilator days in $2009^{(7)}$.

Surgical site infections (SSIs) are those infections that occur in the surgical patient within 30 days of an operation or within 1 year of the operation if an implant was placed. Use of infection control bundles directed at improving compliance with infection control policies is having a dramatic effect on ICU acquired nosocomial infections ${ }^{(8)}$.

Total cost was divided into fixed costs and variable costs. The fixed cost per day per patient was calculated. The variable cost was individually calculated from the costs of routine procedures and also included expenditure on pharmaceuticals, blood products, biochemical, hematological, and bacteriologic tests, radiology, image diagnosis procedures, and other procedures (9)

This study was done to detect the direct cost of infection control measures and the direct cost of hospitalization among patients in Tanta Pediatric Intensive Care Unit in the period between June 2016 and July 2017.

\section{PATIENTS AND METHODS}

A prospective case control study was performed in the Pediatric Intensive Care Unit (PICU) at Tanta University Hospital including patients attending the unit from October 2016 to September 2017. The study was approved by the Ethics Board of Tanta University.

Patients who acquired hospital-acquired infections (HAIs) were considered as cases and patients who did not acquire HAIs were considered as control group.

A total of 50 patients of 1 month to 16 years of age in PICU were categorized into 2 groups: 25 patients who acquired HAIs as a case group and 25 patients who didn't acquire HAIs, matched for age and sex were considered as the control group in this study. Infants or children free from sepsis at the time of admission and acquired an infection in PICU after 48 hours of admission with stay length of 7 days or more were enrolled in this study. Also patients with a known highly infectious disease, surgical patients, patients with regular hemodialysis before admission, trauma patients, post-transplant patients or patients admitted to PICU with infection or discharged before 7 days were excluded.

\section{All children in both groups were subjected} to: (a) Complete history taking: including age, sex, cause of admission, date of admission and past history of previous disease. (b) General and systemic examination: All cases were examined to demonstrate evidence of infection such as fever more than $38^{\circ} \mathrm{C}$, rapid or labored breathing, cool extremities, color changes, tachycardia, mental status changes, hypotension and localizing signs of infection. (c) Routine Laboratory investigation: Complete blood count, qualitative $C$ - reactive protein, renal and liver function tests, arterial blood gases and serum electrolytes. (d) Radiological investigation: $\quad X-\quad$ Rays, ultrasonography, computerized tomography, echocardiography... etc. according to each patient presentation. (e) Bacteriological examination: Blood culture, body fluids culture according to each patient presentation and antibiotic sensitivity. (f) Cost of pediatric 
intensive care unit stay: the variable costs were individually calculated from the costs of routine procedures and also included expenditure or pharmaceuticals, blood products, biochemical, hematological, and bacteriologic tests as well as radiology, image diagnosis procedures, and other procedures. (g) Cost of prevention and control of infection in healthcare: Cost of infection control strategy includes the cost of: 1Standard precautions as: routine hand hygiene, wearing of aprons/gowns, use of face and protective eyewear for procedures, wearing gloves, routine cleaning of surfaces, cleaning of shared clinical equipment and site decontamination after spills of blood or other potentially infectious materials. 2- Transmissionbased precautions as: Contact precautions, droplet precautions and airborne precautions.

The cost in the current study was estimated through direct medical cost which included: Medication, laboratory and radiographic testing, Feeding, Procedures and requirements and infection control measures. The prices used according to the period from October 2016 to September 2017 for calculating patients cost. Also, total infection control cost and infection control cost per day were compared for patients with and without HAIs.

\section{Statistical analysis}

In addition to the descriptive data, statistical analysis was done using IBM SPSS STATISTC VERSION 21 PROGRAM. Quantitative data were described using range (minimum and maximum), mean and standard deviation (SD). Qualitative data were described using number and percent and Chi square (x2) test. $P<0.05$ was accepted as significant.

\section{RESULTS}

Assessments of the measured parameters in the different submitted groups were presented in the following figures:

- Regarding types of HAI, which were detected in cases admitted in PICU included in the current study, $47.06 \%$ showed evidence of BSI, 35.29\% had VAP, while UTI represented $17.65 \%$ of cases as shown in figure (1). As regards organisms isolated, thirty-six cultures were done for patients of the current study with HAI. Klebsiella pneumoniae was the most frequently isolated organism (27.7\%) followed by Coagulase -ve staphylococcus (CoNS) (25\%), Pseudomonas aeruginosa (16.6\%), MRSA (13.8), Escherichia coli (8.3\%), Acinetobacter (5.5\%), and Candida species (2.7\%) as shown in figure (2).

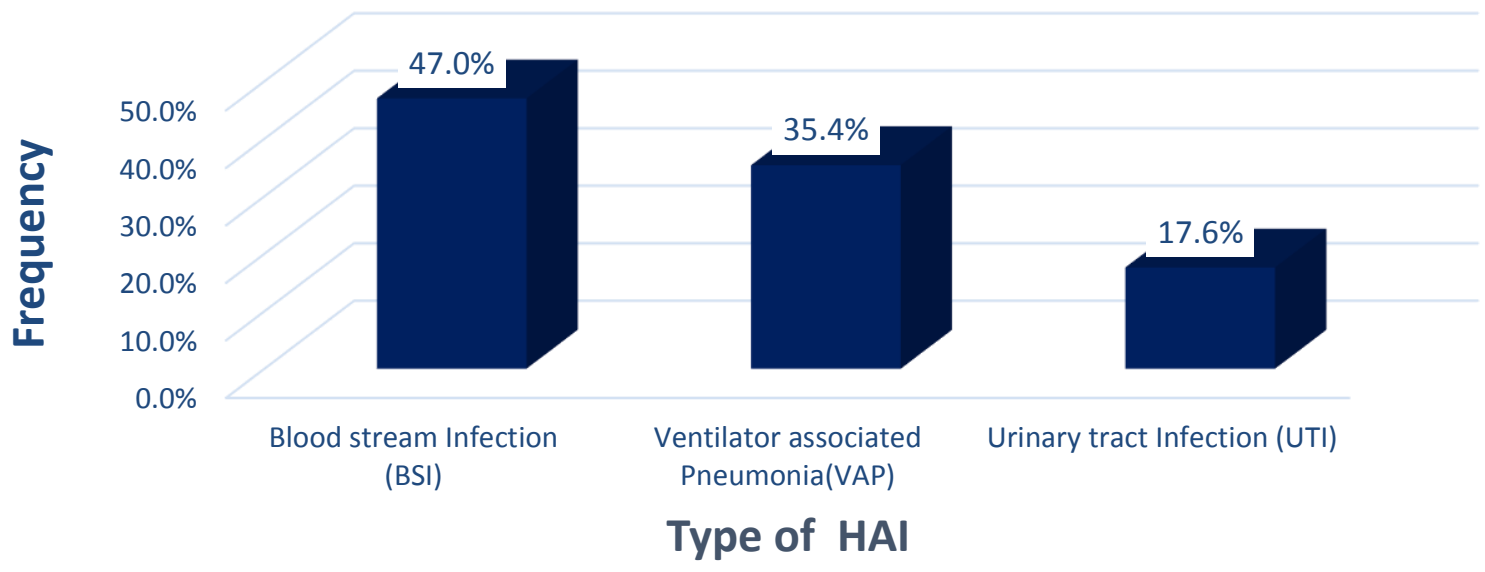

Figure (1): Frequency distribution of the types of HAI among PICU admitted Patients. 


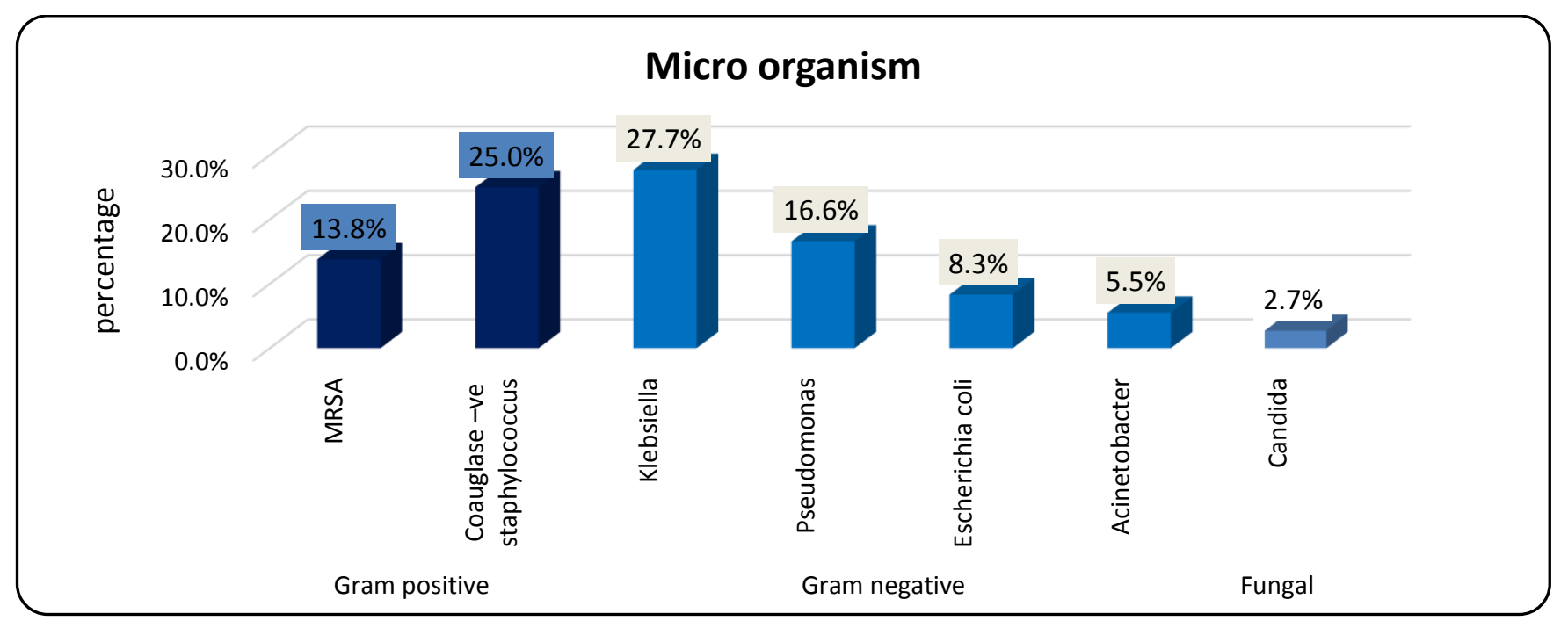

Figure (2): Organisms isolates of cases of HAI in PICU.

- Regarding the invasive procedures for patients included in the current study: The most frequent procedures used in PICU were the central venous catheter (70\%), endotracheal intubation (62\%) and the urinary catheter (36\%). Other procedures were specific to some patients such as chest tube (4\%). $92 \%$ of cases with HAI \& $48 \%$ of cases without HAI were subjected to central venous catheter insertion. While $84 \% \& 40 \%$ of cases with and without HAI respectively needed to endotracheal intubation. $40 \%$ of cases with HAI subjected to urinary catheter insertion but $32 \%$ of cases without HAI needed it and $8 \%$ of cases with HAI underwent chest tube insertion (figure 3).

\section{Procedures}

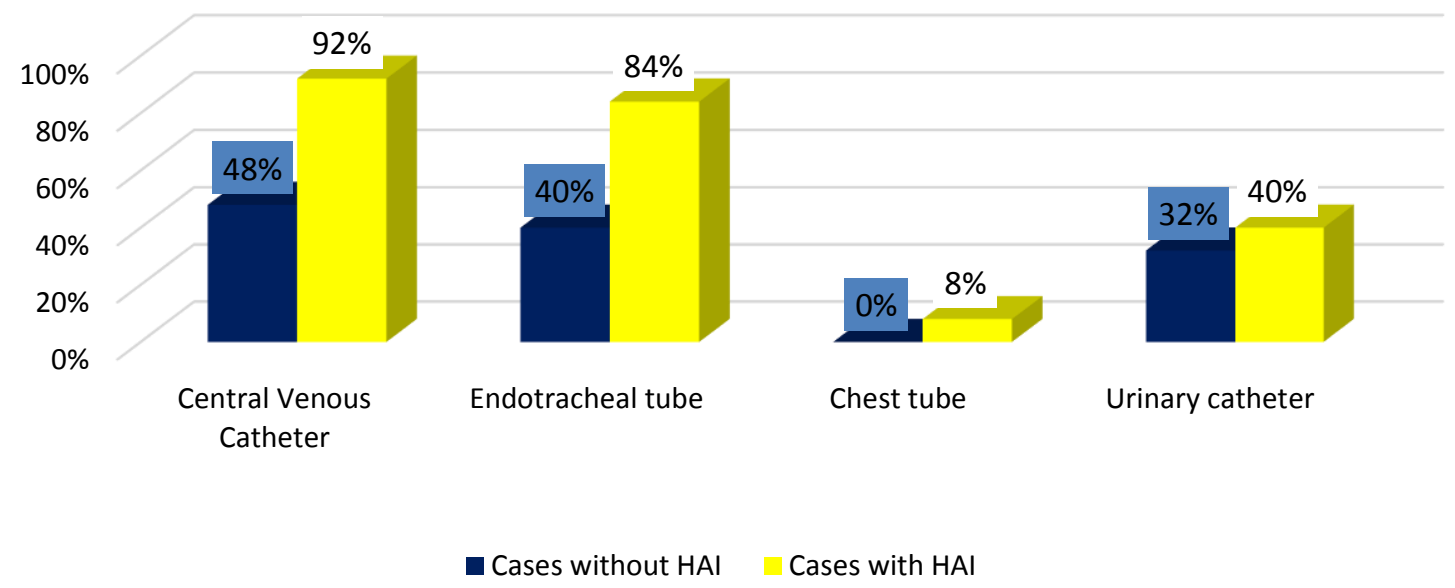

Figure (3): Frequency distribution of the patients with and without HAI admitted to PICU as regards procedures done at the time of admission

- Regarding underlying diseases among cases with HAI, 20\% of cases had congenital heart disease, $16 \%$ had cerebral palsy and epilepsy, $12 \%$ had degenerative brain disease, $8 \%$ had metabolic disorder, bleeding disorders and hypoxic ischemic encephalopathy , $4 \%$ had hydrocephalus, gulliane barrie syndrome and laryngeomalecia as shown in figure (4). 


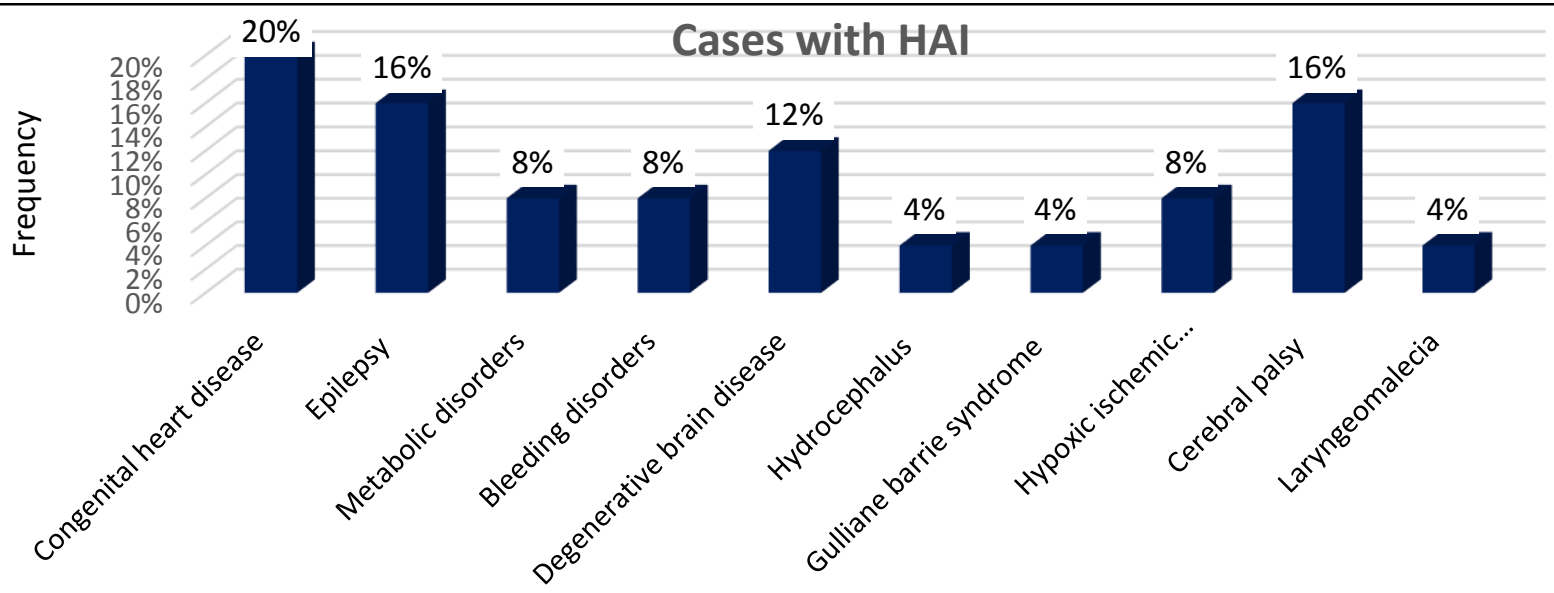

Underlying diseases

Figure (4): Frequency distribution of the patients with HIA admitted to PICU as regards underlying diseases at the time of admission.

- In this study, 22 of our patients (44\%) died during their stay in PICU. The mortality was significantly increased in patients who developed HAI as $68 \%$ of 25 patients with HAI died, where $20 \%$ of 25 patients without HAI died. 1 case (4\%) with HAI \& 11 (44\%) cases without HAI improved and transferred to ward. 6 cases $(24 \%)$ with HAI \& 7 cases $(28 \%)$ without HAI were transferred to intermediate care. Mortality rates were $68 \%$ and $20 \%$ for patients with and without HAI respectively (figure 6).

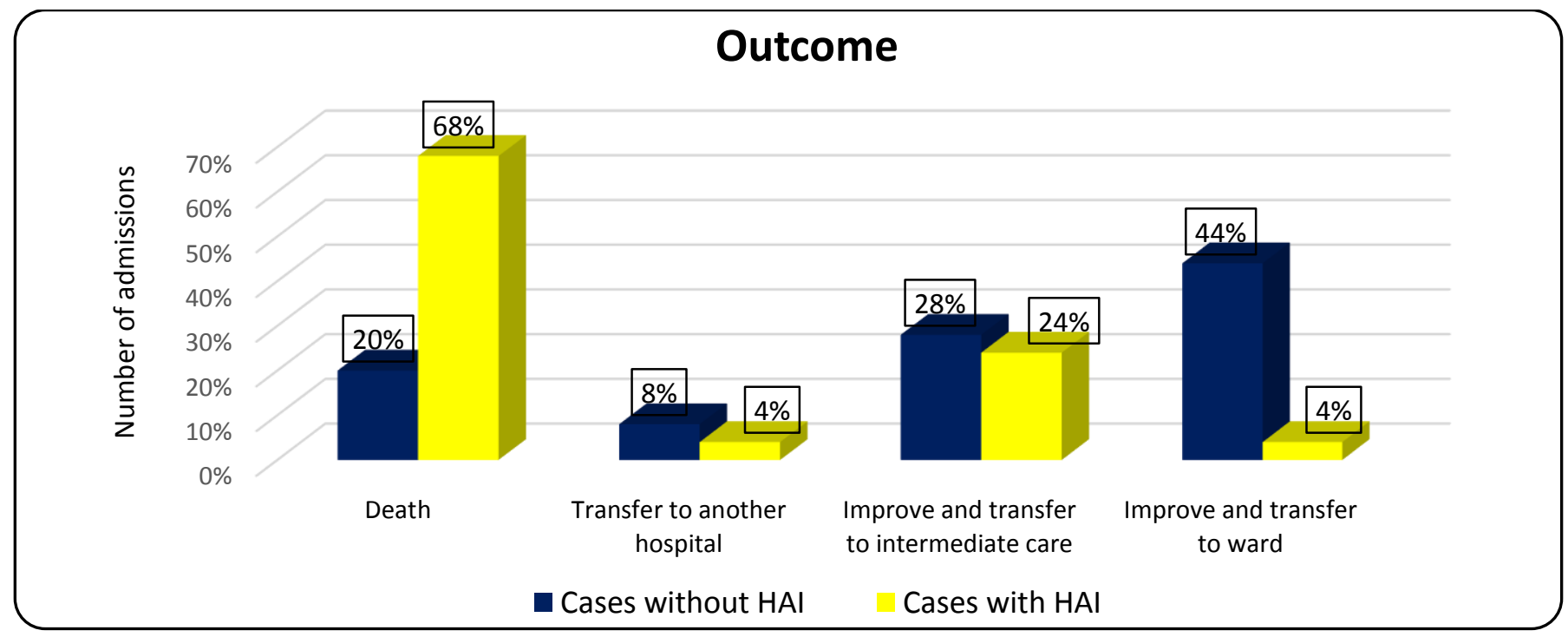

Figure (6): State of discharge of PICU Patients with and without HAI.

- As regards investigations cost in the present study, estimated costs has been based upon prolonged PICU length of stay and variable costs of the different medical procedure. The mean cost of laboratory and radiological testing in patients with HAI were 849.20 E.P \& 254.80 E.P respectively, which were higher than the mean cost of those without HAI (307.12 E.P \& 108.80 E.P respectively) as shown in figure (7). 


\section{Type of investigation}

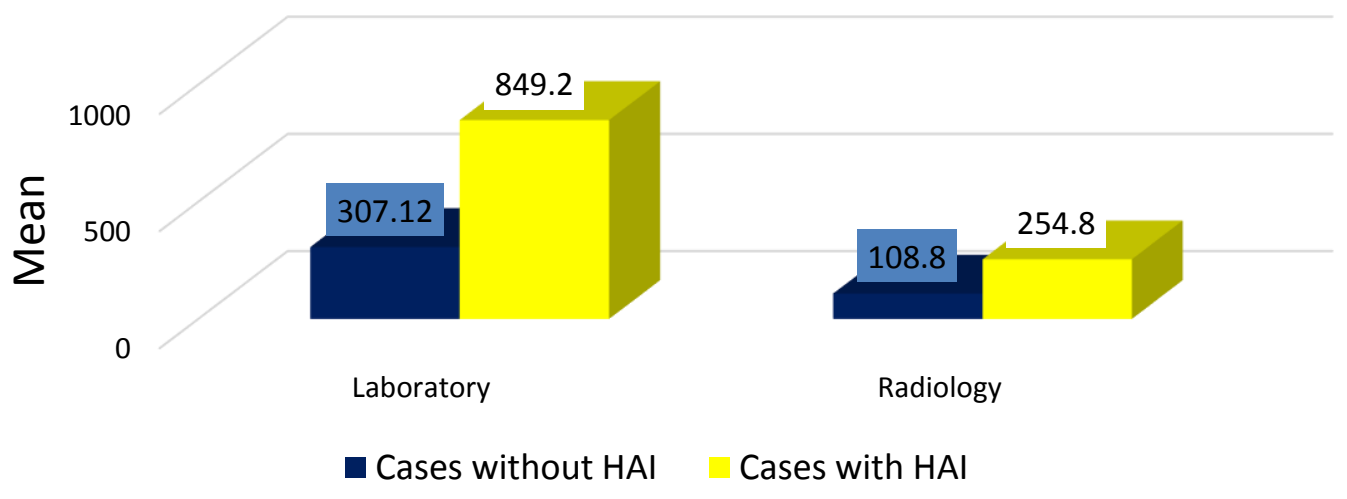

Figure (7): Direct cost in Egyptian Pounds of lab and radiological investigation done to patients of PICU with and without Infection.

- Regarding the mean cost of procedure, requirements, feeding and medication, in PICU for patients included in the current study: It was significantly higher for patients with HAI than patients without HAI, they were $382.52,3364.0884,231.72 \& 1941.44$ E.P respectively, while for patients without HAI, they were $143.44,1174.6892,115.20 \& 342.42$ E.P respectively (figure 8).

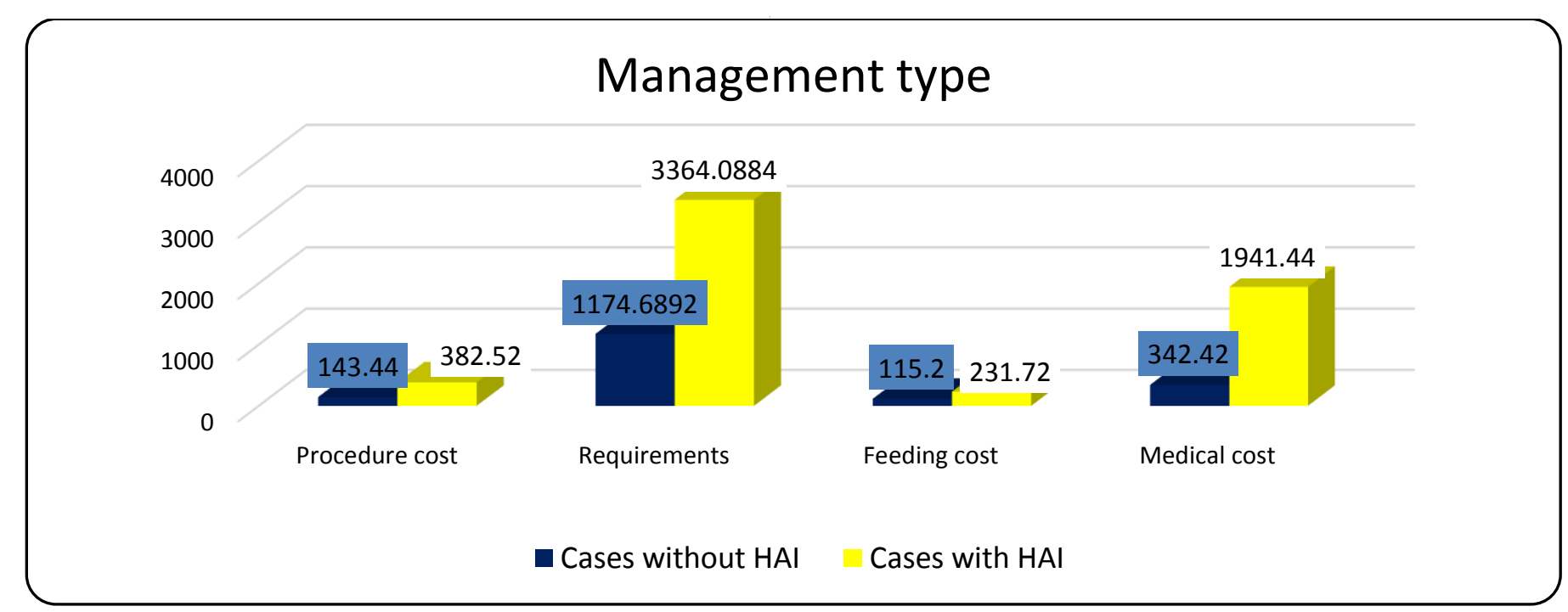

Figure (8): Direct cost [in Egyptian pounds] of the management among patient of PICU with and without HAI.

- As regards the average total direct cost of management in the current study: For cases with HAI, it was 7068.77 E.P, which was significantly higher than that for patients without HAI [2191.66 E.P] as shown in figure (10). Regarding the cost per day in the current study: There was a highly significant increase in the average cost per day between patients with and without HAI. The average cost per day for cases with and without HAI was 381.74 EP \& 300.74 E.P respectively (figure 9). 


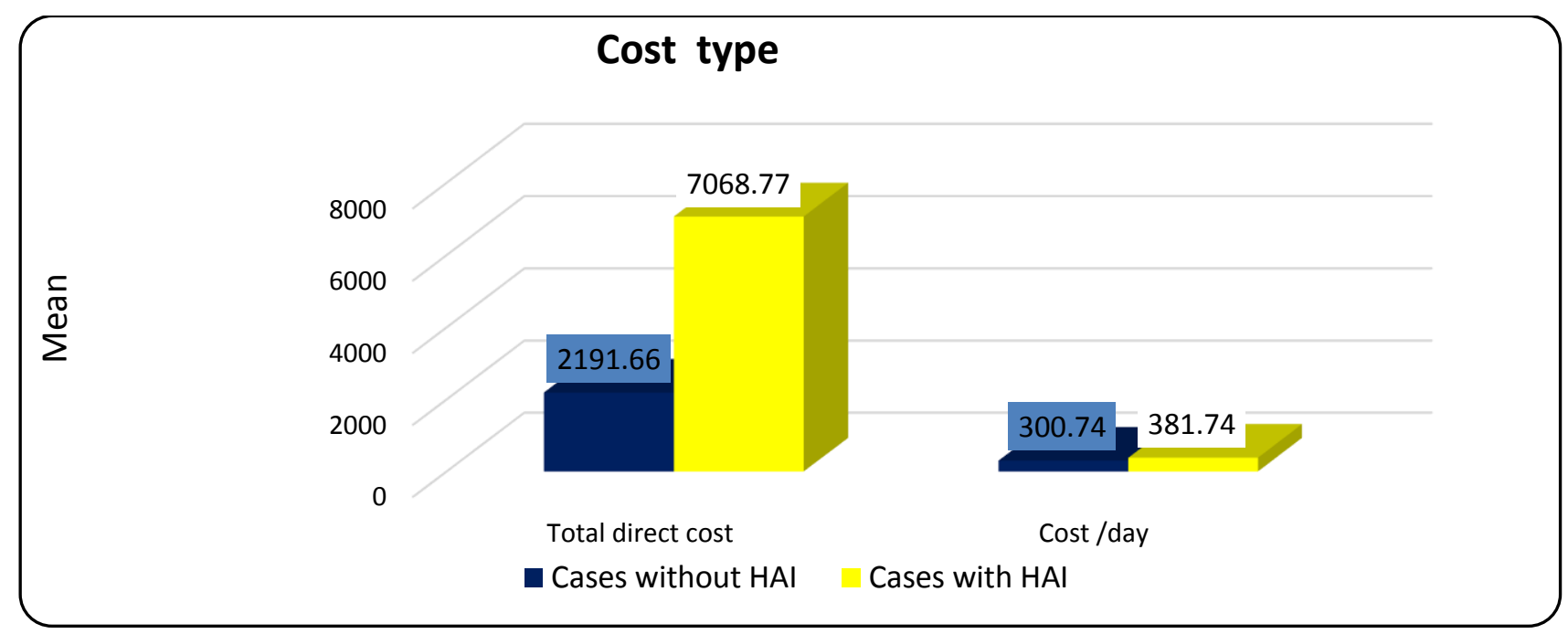

Figure (9): Mean total direct cost [in Egyptian Pounds] of management per stay and mean cost in pounds / day with and without HAI in patients of PICU.

- As regards the mean of the total direct cost of hospitalization: In our study, the mean cost for cases with HAI was 8940.45 E.P, which was significantly higher than that for patients without HAI [2935.63 E.P]. Regarding the cost of hospitalization per day: It was significantly lower in cases without HAI than cases with HAI [400.91 E.P and 480.32 E.P respectively] as shown in figure (10).

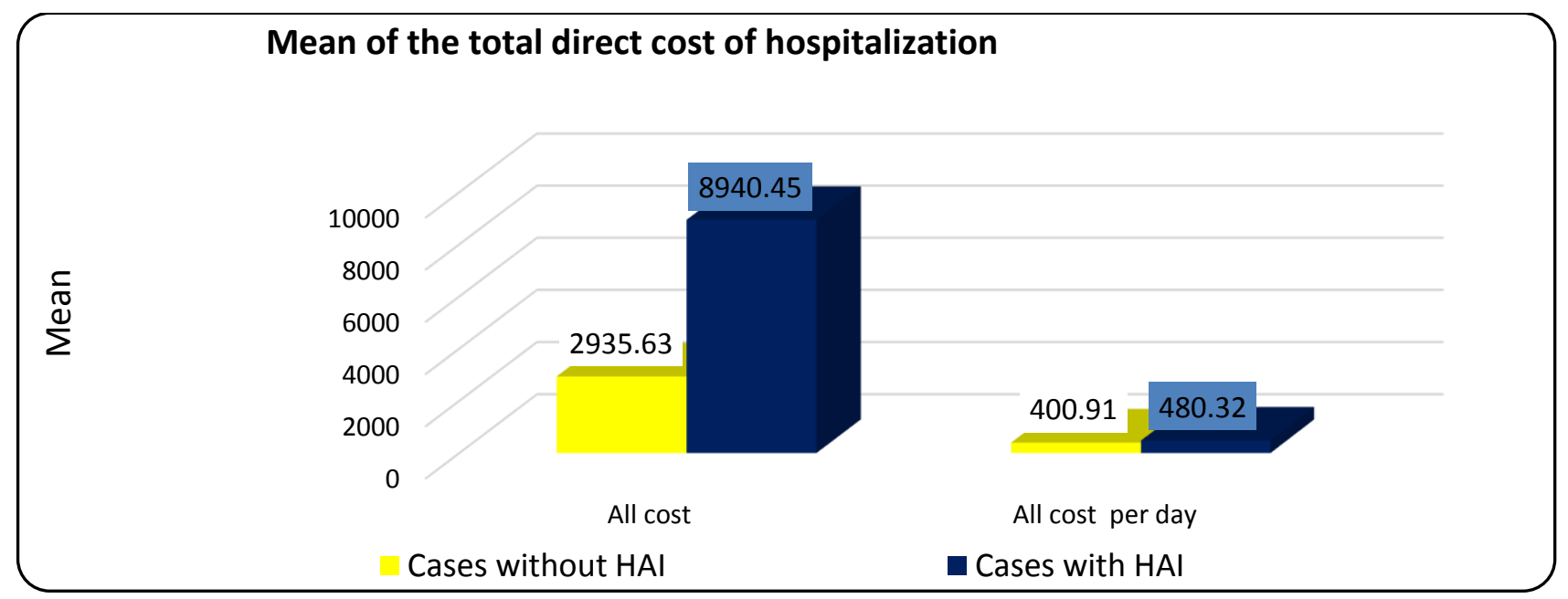

Figure (10): Mean total direct cost [in Egyptian Pounds] of hospitalization and mean cost in pounds / day with and without HAI in patients of PICU.

- As regards the cost of infection control measures in this study: The mean direct costs of gloves, disposable gowns, masks, handling of disposable sharps, waste management, environment cleaning and hand hygiene in cases with HAI per stay were 966.4 \pm 628.63, $572.4 \pm 374.69,120.64 \pm 72.48,30.53 \pm$ $19.98,14.77 \pm 3.122,71.55 \pm 46.84$ and $95.4 \pm 62.45$ E.P respectively. While in cases without HAI they were $384 \pm 82.108,223.2 \pm 47.497,48.04 \pm 11.91,11.90 \pm 2.53,11.87 \pm 0.383,27.75 \pm 5.93$ and 37.2 \pm 7.92 E.P respectively, which were significantly lower than those for cases with HAI per stay (figure 11). 


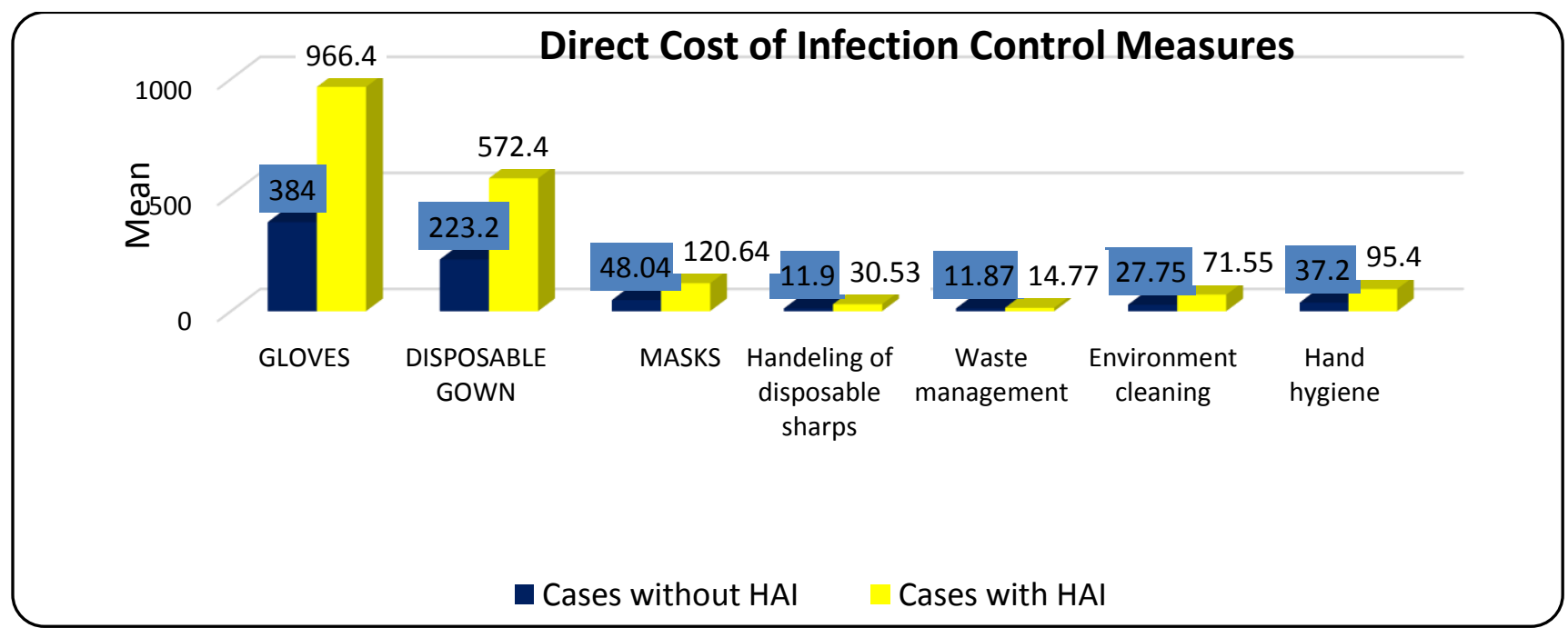

Figure (11): Mean of direct cost of infection control measures [in Egyptian Pounds] per stay used for prevention and control of HAI among patients of PICU.

- As regards the total cost of infection control measures for cases with HAI, it ranged between 701.20 - 5434.60 E.P with a mean of $1871.69 \pm 1207.63$ E.P, wile, it was between 402.40 - 1084.60 E.P, with a mean of $743.96 \pm 154.89$ E.P for cases without HAI that was significantly lower than that for cases without HAI. The daily cost of infection control measures for cases with HAI was ranged between 94.67 - 101.1 E.P, with a mean of $98.58 \pm 1.56$ E.P, while, it was between 93.03 - 106.2 EP with a mean of $100.17 \pm 3.50$ E.P for cases without HAI. There was no significant difference between cases with and without HAI as regard daily cost of infection control measures (figure 12).

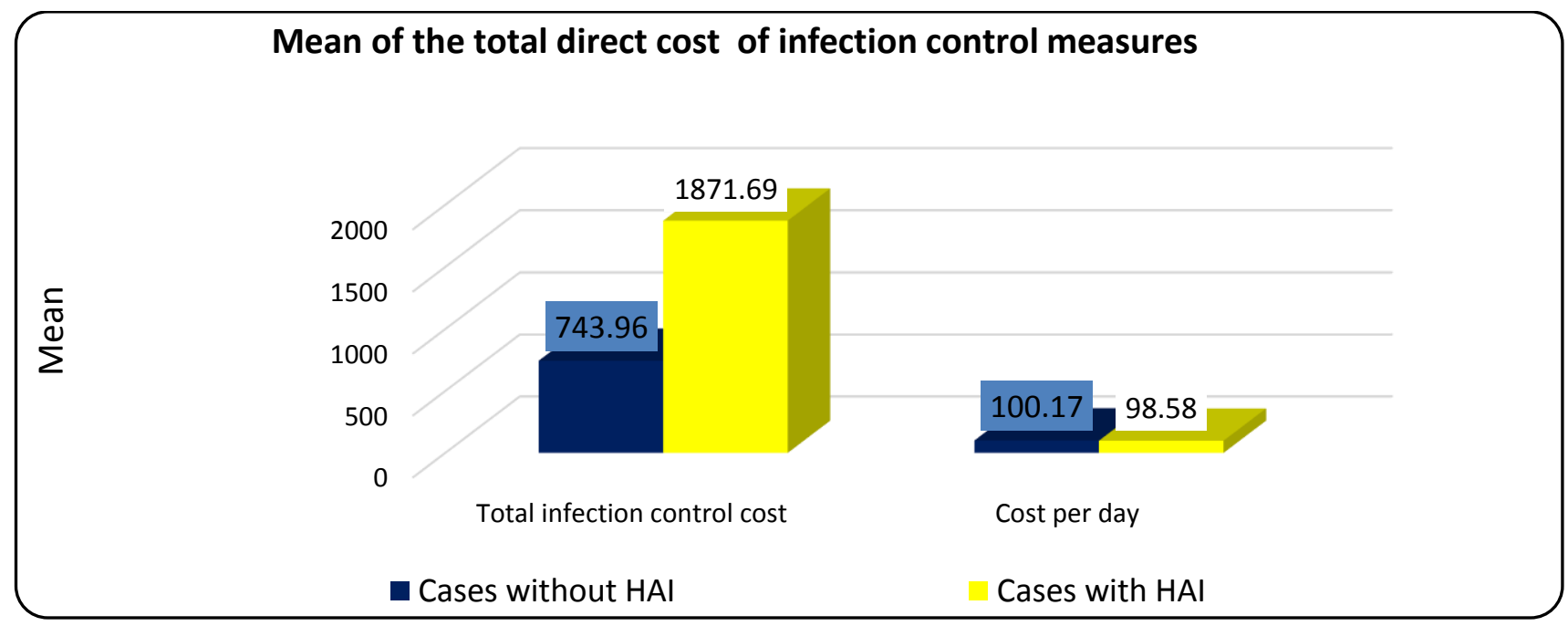

Figure (12): Mean total direct cost of infection control measures [in Egyptian Pounds] per stay used for prevention and control of HAI among patients of PICU.

- Regarding the length of stay in the present study, the mean length of stay (LOS) in PICU was 19.08 days [range 7-56] for patients with HAI, while in patients without HAI it was 7.44 days [range 4-11] as shown in figure (13). 


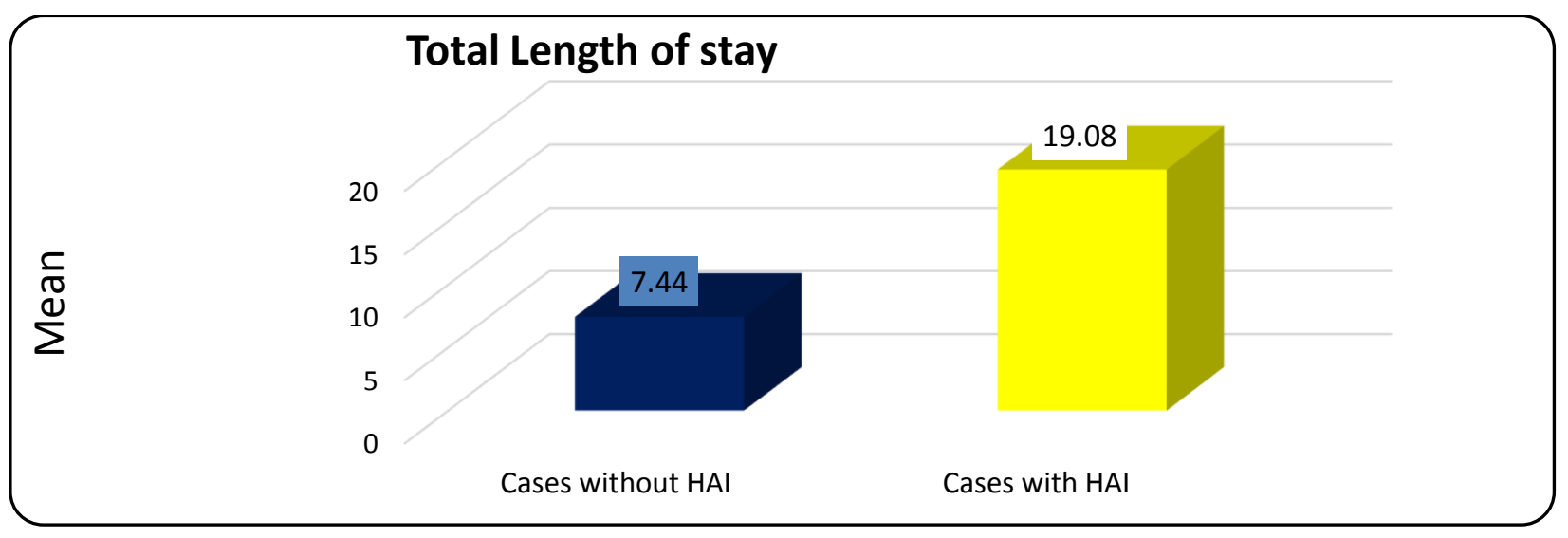

Figure (13): Total length of hospital stay [in days] of PICU in patients with and without HAI.

\section{Discussion}

Healthcare-associated infections has a significant and largely avoidable economic impact at both the patient and population levels, including out-of-pocket costs to patients and costs incurred through lost productivity due to morbidity and mortality. According to the US Centers for Disease Control and Prevention, the overall annual direct medical costs of HAI to hospitals in the USA alone ranges from US\$ 35.7 to 45 billion, while the annual economic impact in Europe is as high as $€ 7$ billion ${ }^{(10)}$.

In agreement with the current findings a previous study in Tanta Pediatric Intensive Care Unit done by Atia, ${ }^{(11)}$ who concluded that klebsiella pneumoniae was the most frequently isolated (30.4\%) followed by acinetobacter $(26.09 \%)$, coagulase negative staphylococci (13.04\%), S. aureus (8.7\%), pseudomonas aeruginosa $(8.7 \%)$ and candida species $8.7 \%$. but it showed that VAP was the most frequent infection in the PICU $(52.3 \%)$ followed by BSI (33.8\%), UTI (7.7\%) and skin infections $(6.2 \%)$ with is against the current study findings . Ibrahim ${ }^{(12)}$ reported that in HAI most common infections were respiratory $(53.3 \%)$ followed by UTI (20\%) then BSI $(11.7 \%)$ which is also is against the current study findings. Another disagreement is the
Egyptian study by Rasslan et al. ${ }^{(13)}$ reported that the VAP rate was 31.8 per 1000 mechanical ventilation-days in the PICUs. Cultures were performed for VAP patients where $87.2 \%$ showed growth. Klebsiella and methicillin-resistant Staphylococcus aureus (MRSA) were the most common microorganisms associated with VAP, followed by Pseudomonas aeruginosa. The BSI rate was 18.8 per 1000 central line-days in the PICUs. The UTI rate per 1000 UC-days was 0.0 in the PICUs.

In agreement with the present study is the one that was done in Mansoura University Pediatric Hospital (14) and reported that Klebsiella was the most common isolate (19.1\%) followed by Staphylococcus aureus (12.2\%), Candida spp. (9.8\%), MRSA (6.5\%).

In contrast to the current study van vught et al. ${ }^{(15)}$ reported that Pneumonia was the most common ICU acquired infection in 117 patients [48.4\%], Catheter-related bloodstream infection 61 patients $(16.7 \%)$ and Urinary tract infections 6 patients (1.6\%). They also coincides with the present study in reporting that $97.4 \%$ cases with ICU acquired infections $\& 94.5 \%$ without ICU acquired infections subjected to urinary catheter. 
While, central venous catheter $93.5 \%$ in cases with ICU acquired infections and without ICU acquired infections $79.4 \%$. (99.1\%) cases with ICU acquired infections \& without ICU acquired infections (92\%) subjected to endotracheal intubation. Finally they reported that the median length of stay in cases with HAI was 22 ranged (15-33) and for cases without HAI was 5 ranged (3-9).

In agreement with the present study Becerra et al. ${ }^{(16)}$ reported that frequency of BSI was $20 \%$ then VAP was $8.6 \%$ and at last $3.6 \%$ for UTI. They also reported that the admitted children, $43 \%$ had a CVC, $70 \%$ ETT and $38 \%$ UC. Also, in $59 \%$ of cases of BSI, at least one microorganism was isolated in blood cultures. But by contrast they showed that the most common was Candida $(41 \%$ of isolations). In $97 \%$ of VAPs, at least one microorganism was isolated from tracheal aspirate. Pseudomonas (52\%) was the most common isolate. In $86 \%$ of UTI, at least one microorganism was isolated. Candida was isolated in 5/7 cases. Finally they reported that $31(38.3 \%)$ with HAI $(\mathrm{n}=81)$ died, $74(20.4 \%)$ without HAI $(\mathrm{n}=363)$ died. Another study done by Ašembergiene et al. ${ }^{(17)}$ recorded that the mortality rate was $7.7 \%$ for patients with HAI and $2.7 \%$ for patients without HAI. The mean length of stay, days, for patients with HAI, was 11.7 days and 5.2 days for those without HAI.

Also in contrast to the present work Abramczyk et al. ${ }^{(18)}$ showed that the HAI incidence was $18.3 \%$ and the mean infection rate per 1,000 patient days was 46.1 ; the ventilator-associated pneumonia rate was 18.7 per 1,000 ventilator days; the central lineassociated bloodstream infection rate was 10.2 per1, 000 central line days; and the urinary tract catheter-associated infection rate was 1.8 per 1,000 catheter days. Pneumonia was the most common NI (31.6\%) followed by bloodstream infections (17.3\%) and surgical site infection (17.3\%). They also agreed to the current work and reported that the mean length of stay with HAI was 15.7 and the mean for cases without HAI was 4.1. In addition they reported that the mortality rate for cases with and without HAI was $28.3 \%$ \& $14.6 \%$ respectively.

Concordantly Urrea et al. ${ }^{(19)}$ reported that the most frequent type of nosocomial infections were BSI (51.7\%), followed by respiratory infection (19\%) and UTI (17.2\%). They also disagreed to the current work and reported that the most common microorganisms isolated were coagulasenegative staphylococci $(38.8 \%)$, followed by pseudomonas aeruginosa (23.6\%), (9.7\%) and (2.8\%) for Escherichia coli and Klebsiella spp., respectively. Regarding the invasive procedures, the most frequent procedures used in PICU were the central venous catheter (70\%), endotracheal intubation (62\%), and the urinary catheter $(36 \%)$. Other procedures were specific to some patients such as chest tube (4\%). $92 \%$ of cases with HAI \& $48 \%$ of cases without HAI were subjected to central venous catheter insertion and this coincides with the current results. but $84 \%$ \& $40 \%$ of cases with and without HAI needed endotracheal intubation respectively. $40 \%$ of cases with HAI subjected to urinary catheter insertion but $32 \%$ of cases without HAI needed it and $8 \%$ of cases with HAI underwent chest tube insertion. A study done by Morillo-Garcia et al. ${ }^{(20)}$ reported that most invasive procedures used were CVC \& ETT 64\% and 55\% respectively and this coincides with the present results.

Regarding the length of stay, in the present study, the mean length of stay (LOS) in PICU was 19.08 days (range 7-56) for patients with HAI and median length of stay was 15 , while in patients without HAI was 7.44 days (range 4-11) and median was 7. In 
addition, they reported that the average Imaging test cost in cases with and without HAI was $143.98 € \& 63.67 €$ respectively. The average laboratory cost of cases with and without HAI was $240.49 € \& 83.25 €$. The mean feeding cost of patients with and without HAI was $20.85 € \& 15.34 €$ respectively. Mean procedures cost of patients with and without HAI were $111.75 € \& 33.67 €$ respectively. Besides, the average total cost of management in cases with HAI $28406.39 €$ that was significantly higher than that for patients without HAI 3773.26. Finally, the average total cost in cases with HAI was $35507.99 €$, which was significantly higher than that for patients without HAI (4716.58 €).

Di GIOVINE et al. ${ }^{(21)}$ also agreed with the current results and reported that primary nosocomial bloodstream infection (BSI) is a common occurrence in the intensive care unit (ICU). Coagulase-negative and Staphylococci were the most common isolate $(45.6 \%)$, followed by Staphylococcus aureus (10.3\%) and candida (7.4\%). In addition, they reported that total cost of patients with HAI was 60,650 $\$$ that was higher than that for patients without HIA $(36,899$ \$).

In a study done in England by Plowman et al. ${ }^{(22)}$ did not match the present findings by showing that the incidence of HAI was urinary tract infections in 107 patients $(2.7 \%)$, then lower respiratory tract infections 48 patients (1.2\%) and bloodstream infections 4 patients $(0.1 \%)$. Moreover, they reported that mean procedure cost in patients with and without HAI was $118.35 £ \& 9.95 £$ respectively. Finally, they reported that mean total cost of management in patients with and without HAI was $4782.03 £ \& 1628.38 £$ respectively which coincides with the present results.

In agreement with the present work; Thoa et al. ${ }^{(23)}$ reported that the mean cost of laboratory and radiology investigations in patients with HAI was 49.3 \$ \& 307.4 \$ respectively that was higher than mean cost of those without HAI (11.6 \$ \& 179.8 \$). In addition, their study showed that the mean cost of medication in patients with HAI was 1113.6 \$ that was higher than mean cost of those without HAI (359.6 \$). For the cost effectiveness of a hand hygiene program on healthcare-associated infections in intensive care patients at a tertiary care hospital in 2 intensive care and 15 critical care units, they reported that the mean cost for patients with an HAI overall was $\$ 1,908$, which is 2.5 times higher than the costs for patients without an HAI (\$777). The incidence of HAIs decreased from $31.7 \%$ in the pre-intervention group to $20.3 \%$ in the post intervention group. Therefore, the effectiveness of the hand sanitizer program was $36 \%$, resulting in 11.4 HAIs prevented per 100 patients in the prevention group. The cost-effectiveness was estimated at \$1,074 per HAI prevented. A study in the USA by Elward et al. ${ }^{\text {(24) }}$ reported that the mean laboratory cost in cases with BSI and without BSI was $(2333$ \$ \& 2190 \$ respectively) which is matching to the present work while the mean radiology cost in cases with and without BSI was $(601 \$$ \& 646 \$ respectively) which is against the current findings. Concordantly, they also reported that the mean direct total cost of PICU admission was 70936 \& for infected patients and 10828 \$ for uninfected patients.

Coincidently with the current work Kostakoglu et al. ${ }^{(25)}$ reported that the mean cost of medication in patients with HAI was 3495 \$ that was higher than mean cost of those without HAI (2232\$). They also reported that the mean total cost in patients with HAI was 5439 \$ that was higher than mean cost of those without HAI (3446\$).

In agreement with current results Warren et al. ${ }^{(26)}$ reported that the mean total hospital 
cost for patients with and without VAP was 70,568 \$ \& 21,620 \$ respectively. Also, Alrawajfah et al. ${ }^{(27)}$ reported that the mean total charges for patients with BSIs were $85,813 \$$ compared to $22,821 \$$ for uninfected patients.

$$
\text { Concordantly, Conterno et al. }
$$

reported that the total cost of the infection control intervention to reduce nosocomial extended-spectrum b-lactamase (ESBL) transmission in a non-outbreak setting was \$ 552186.50 over four years, for a mean cost of $\$ 138046.63$ per year, and \$ 3191.83 per case admitted. The use of a private room had the greatest cost impact, accounting for $85 \%$ of the total cost, followed by the cost of supplies for contact precautions (7.8\%) and additional nursing time $(6.5 \%)$. Also, Jarvis ${ }^{(29)}$ reported that even a minimally effective infection control program that prevented only $6 \%$ of all nosocomial infections would offset the $\$$ 60,000 estimated annual cost of the infection control program. If the infection control program was to prevent $32 \%$ to $50 \%$ of the infections, the infection control program

\section{REFERENCES}

1- Sodhi J, Satpathy S, Sharma D et al. (2016): Healthcare associated infections in Paediatric Intensive Care Unit of a tertiary care hospital in India: Hospital stay \& extra costs. Indian J Med Res., 143 (4): 502.

2- Brindha S, Jayashree M, Singhi S et al. (2010): Study of nosocomial urinary tract infections in a pediatric intensive care unit. J Trop Pediatr., 57 (5): 357-62.

3- National Health, Medical Research Council (2010): Australian guidelines for the prevention and control of infection in healthcare. Available at: https://www.nhmrc.gov.au/guidelinespublications/cd33. Accessed (July 2017).

4- Centers for Disease Control and Prevention (2016): https://www.cdc.gov/

5- Banerjee S, Grohskopf L, Sinkowitz-Cochran R et al. (2006): Incidence of pediatric and neonatal intensive care unit-acquired infections. Infect Control Hosp Epidemiol.. 27(6):561-70.

6- Elward A, Warren D, Fraser V (2002): Ventilator-associated pneumonia in pediatric would result in net savings of $\$ 260,000$ to $\$$ 440,000 .

Chaix et al. ${ }^{(30)}$ agreed to the present work and reported that the mean cost of medication in patients with HAI was 9550 \$ that was higher than mean cost of those without HAI (6040\$). As regards the average total direct cost of management in our study, it was 7068.77 E.P for cases with HAI, which was significantly higher than that for patients without HAI [2191.66 E.P]. They also reported that the mean total cost of management in patients with HAI was 30225 \$ that was higher than the mean cost of those without HAI (20950\$).

Conclusion: The current study showed that the average total cost of infection control measures for cases with HAI was 1871.69 E.P, which was significantly higher than that of cases without HAI. The cost of infection control is much lower than the cost of hospitalacquired infections management that prolonged hospital stay causing financial losses to the health system.

intensive care unit patients: risk factors and outcomes. Pediatrics, 109(5):758-64.

7- Dudeck M, Horan T, Peterson K et al. (2011): National Healthcare Safety Network (NHSN) Report, data summary for 2010, device-associated module. Am J Infect Control., 39(10):798-816.

8- Reade E, Talbott G, Rowin M (2014): HospitalAcquired Infections and the Pediatric Intensive Care Unit. In: Pediatric Critical Care Medicine. Springer.

9- Garcia S, Ruza F, Alvarado F et al. (1997): Analysis of costs in a pediatric ICU. Intensive Care Med., 23(2):218-25.

10- Storr J, Twyman A, Zingg W et al. (2017): Core components for effective infection prevention and control programmes: new WHO evidence-based recommendations. Antimicrob Resist Infect Control., 6(1):6.

11- Attia A, Abo Elezz A, Hassan A et al. (2015): Hospital Acquired Infections in Pediatric Intensive Care Unit at Tanta University Hospital. M.Sc. Thesis, pediatric, Tanta University, Faculty of Medicine. 
12- Ibrahim Sh (2014): hospital acquired sepsis in Tanta Pediatric Intensive care Unit. M.Sc. Thesis, Pediatric, Tanta University, Faculty of Medicine.

13- Rasslan O, Seliem $Z$, Ghazi I et al. (2012): Device-associated infection rates in adult and pediatric intensive care units of hospitals in Egypt. International Nosocomial Infection Control Consortium (INICC) findings. J Infect Public Health, 5(6):394-402.

14- El-Bayoumi M, El-Nady G, Badr RI (2006): Clinical and microbiological study of nosocomial infection in pediatric intensive care unit (PICU) in Mansoura University Children's Hospital. Egypt J of Med Microb.. 15: 493-503.

15- Van Vught L, Klouwenberg P, Spitoni CR et al. (2016): Incidence, risk factors, and attributable mortality of secondary infections in the intensive care unit after admission for sepsis. Jama.., 315(14):1469-79.

16- Becerra M, Tantaleán J, Suárez V et al. (2010): Epidemiologic surveillance of nosocomial infections in a Pediatric Intensive Care Unit of a developing country. BMC Pediatr., 10(1):66.

17- Ašembergiene J, Gurskis V, Kevalas $\mathbf{R}$ et al. (2009): Nosocomial infections in the pediatric intensive care units in Lithuania. Med., 45(1):29_ 36.

18- Abramczyk M, Carvalho W, Carvalho E et al. (2003): Nosocomial infection in a pediatric intensive care unit in a developing country. Brazilian J Infect Dis., 7(6):375-80.

19- Urrea M, Pons M, Serra M et al. (2003): Prospective incidence study of nosocomial infections in a pediatric intensive care unit. Pediatr Infect Dis J., 22(6):490-3.

20- Morillo-Garcia Á, Aldana-Espinal J, De LabryLima A et al. (2015): Hospital costs associated with nosocomial infections in a pediatric intensive care unit. Gac Sanit., 29(4):282-7.

21- Digiovine B, Chenoweth C, Watts C et al. (1999): The attributable mortality and costs of primary nosocomial bloodstream infections in the intensive care unit. Am J Respir Crit Care Med., 160(3):97681 .
22- Plowman R, Graves N, Griffin M et al. (2001): The rate and cost of hospital-acquired infections occurring in patients admitted to selected specialties of a district general hospital in England and the national burden imposed. J Hosp Infect., 47(3): 198-209.

23- Thoa V, Van Trang D, Tien N et al. (2015): Cost effectiveness of a hand hygiene program on health care--associated infections in intensive care patients at a tertiary care hospital in Vietnam. Am J Infect Control., 43(12):e93--e99.

24- Elward A, Hollenbeak C, Warren D et al. (2005): Attributable cost of nosocomial primary bloodstream infection in pediatric intensive care unit patients. Pediatrics, 115(4):868-72.

25- Kostakoglu U, Saylan S, Karatas M et al. (2016): Cost analysis and evaluation of nosocomial infections in intensive care units. Turkish J Med Sci., 46(5):1385-92.

26- Warren D, Shukla S, Olsen M et al. (2003): Outcome and attributable cost of ventilatorassociated pneumonia among intensive care unit patients in a suburban medical center. Crit Care Med., 31(5):1312-7.

27- Al-rawajfah O, Hewitt J, Stetzer F et al. (2012): Length of stay and charges associated with health care-acquired bloodstream infections. Am J Infect Control,40(3):227-32.

28- Conterno L, Shymanski J, Ramotar $\mathrm{K}$ et al. (2007): Impact and cost of infection control measures to reduce nosocomial transmission of extendedspectrum $\quad \beta$-lactamase-producing organisms in a non-outbreak setting. J Hosp Infect., 65(4):354-60.

29- Jarvis WR (1996): Selected aspects of the socioeconomic impact of nosocomial infections: morbidity, mortality, cost, and prevention. Infect Control Hosp Epidemiol.. 17(8):552-7.

30- Chaix C, Durand-Zaleski I, Alberti C et al. (1999): Control of endemic methicillin-resistant Staphylococcus aureus: a cost-benefit analysis in an intensive care unit. Jama., 282(18):1745-51. 